\title{
Impacto del cigarrillo en el tracto gastrointestinal: efecto diferencial en enfermedad de Crohn y colitis ulcerosa
}

\author{
Loni Berkowitz ${ }^{1}$, Manuel Álvarez-Lobos ${ }^{1}$
}

\begin{abstract}
Resumen: El impacto del consumo de cigarrillo a nivel respiratorio y cardiovascular ha sido extensamente investigado, sin embargo, aún queda mucho por estudiar sobre su impacto en el tracto gastrointestinal. Está demostrado que el tabaquismo es un importante factor de riesgo para desórdenes gastrointestinales, incluyendo úlceras pépticas, Enfermedad de Crohn y distintos tipos de cáncer. Los mecanismos propuestos incluyen daños a nivel de la mucosa, alteraciones en la irrigación del tejido y cambios en la respuesta inmune. Paradojalmente, el consumo de cigarrillo constituye un factor protector para el desarrollo y la progresión de la Colitis Ulcerosa. Esta patología, junto a la Enfermedad de Crohn, conforman las dos entidades más importantes dentro de las Enfermedades Inflamatorias Intestinales y comparten diversas características. El impacto diferencial del consumo de cigarrillo entre ambas ha sido un tema de gran interés en los últimos treinta años, y aún no ha logrado ser esclarecido. En esta revisión describiremos los efectos conocidos del tabaquismo sobre el tracto gastrointestinal, y particularmente en la inflamación intestinal, profundizando en los estudios disponibles que intentan explicar el efecto opuesto del cigarrillo en la Enfermedad de Crohn y la Colitis Ulcerosa.
\end{abstract}

Palabras clave: cigarrillo; inflamación gastrointestinal; Enfermedad Inflamatoria Intestinal; Colitis Ulcerosa; Enfermedad de Crohn.

Abstract: The impact of cigarette smoking on respiratory and cardiovascular diseases has been extensively researched, although further investigation is required to elucidate its impact on the gastrointestinal tract. It has been demonstrated that smoking is a major risk factor for gastrointestinal disorders, including peptic ulcers, Crohn's disease and various cancers. Proposed mechanisms include mucosal damage, changes in gut irrigation and changes in the mucosal immune response. Paradoxically, cigarette smoking is a protective factor for the development and progression of Ulcerative Colitis. Both Ulcerative colitis and Crohn's disease represent the two most important entities in Inflammatory Bowel Diseases and share several characteristics. The differential impact of smoking between these two disorders has been a topic of great interest in the last thirty years, and has yet to be clarified. In this review we describe the known effects of smoking on the gastrointestinal tract, and particularly in the intestinal inflammation, delving into the available studies that attempt to explain the opposite effect of smoking in Crohn's disease and Ulcerative colitis.

Keywords: Cigarette; Gastrointestinal inflammation; Inflammatory Bowel Disease; Ulcerative colitis; Crohn's disease.

Fecha de envío: 27 de octubre de 2016 - Fecha de aceptación: 20 de marzo de 2017

\section{Introducción}

Las enfermedades asociadas al consumo de cigarrillo constituyen la causa principal de muertes prevenibles y de disfunciones respiratorias a nivel mundial, significando aproximadamente 6 millones de decesos anuales (Organización Mundial de la Salud, 2015). Las proyecciones sugieren que para el año 2030, el tabaquismo cobrará aproximadamente 10 millones de vidas anuales, siendo un 70\% de estas en países desarrollados (Calafat et al., 2004)tar, and carbon monoxide (CO, donde la tasa de fumadores alcanza aproximadamente un $37 \%$ en hombres y $21 \%$ en mujeres (van Zyl-Smit et al., 2010).
El consumo de cigarrillo constituye un riesgo importante para el desarrollo de enfermedades vasculares, incluyendo aterosclerosis e hipertensión pulmonar (Csordas \& Bernhard, 2013). Sin embargo, la morbilidad y la mortalidad asociada al tabaquismo no se limita únicamente a enfermedades respiratorias y cardiovasculares, sino también envuelve otras enfermedades, incluyendo diversos desórdenes inflamatorios y carcinogénicos. Esto se debe a la gran cantidad de componentes tóxicos presentes en el humo de cigarrillo, cuyo impacto a nivel respiratorio y vascular ha sido extensamente investigado. Sin embargo, el modo por el cual el tabaquismo afecta el tracto gastrointestinal, y particularmente

(1) Departamento de Gastroenterología, Pontificia Universidad Católica, Santiago, Chile

Autor de correspondencia: loniberko@gmail.com 
a la Enfermedad Inflamatoria Intestinal, aún no ha podido ser esclarecido. En esta revisión nos centraremos en el impacto del cigarrillo a nivel gastrointestinal, profundizando en los estudios recientes que intentan explicar su efecto diferencial entre las dos entidades más importantes de Enfermedad Inflamatoria Intestinal, la Enfermedad de Crohn y la Colitis Ulcerosa.

\section{Efectos nocivos del cigarrillo}

El tabaco, principal constituyente de los cigarrillos, es un producto natural formado por diversas moléculas que al fumar son sometidas a altas temperaturas y concentraciones variables de oxígeno, generando una combustión incompleta y originando una gran cantidad de componentes nocivos. Dentro de estos componentes están incluidos diversos carcinógenos (ej., benzopirenos y nitrosaminas), toxinas (ej., nicotina y CO), partículas reactivas (ej., metales pesados) y oxidantes (ej., anión superóxido y aldehídos) (Marcilla et al., 2012).

La corriente principal del humo de cigarrillo, que es ingerida directamente por el fumador, está compuesta por una fase particulada y una fase gaseosa. Los componentes de bajo peso molecular, como el monóxido de carbono y los aldehídos livianos, son los constituyentes principales de la fase gaseosa, la cual pasa rápidamente a la circulación pulmonar (Jaimes et al., 2004). Por otro lado, la nicotina, los compuestos policíclicos aromáticos, las nitrosaminas y los metales pesados, se encuentran predominantemente en el material particulado, el cual podrá ser absorbido por las membranas de las mucosas, piel, alvéolos, y a través del sistema gastrointestinal (Wu \& Cho, 2004).

La inhalación crónica de los más de 7000 componentes tóxicos presentes en el humo del cigarrillo alteran la proliferación celular, la función endotelial (Csordas \& Bernhard, 2013) y la respuesta inmune (Brusselle et al., 2011). Está demostrado que el tabaquismo es capaz de aumentar el riesgo de enfermedades pulmonares obstructivas crónicas, enfermedades cardiovasculares, infecciones, y diversos tipos de cánceres, principalmente, cáncer de pulmón, orofaringe y laringe. De hecho, aproximadamente un 15\% de los casos de cáncer a nivel mundial puedes ser atribuidos al consumo de cigarrillo, siendo el cáncer de pulmón el de mayor correlación con el hábito tabáquico (Yu et al., 2016). Hasta el día de hoy, más de 60 componentes del humo de cigarrillo han sido identificados como carcinógenos y mutagénicos, destacándose algunos hidrocarburos aromáticos policíclicos y las N-nitrosaminas (Organización Mundial de la Salud, 2015). Por otro lado, el humo de cigarrillo posee altas concentraciones de especies reactivas de oxígeno, peroxinitritos, peroxinitratos, radicales libres y compuestos orgánicos reactivos que generan estrés oxidativo y alteran la función endotelial dependiente de óxido nítrico. Las evidencias sugieren que esta fracción oxidante sería el principal estímulo para la formación de placas arteroscleróticas, trombos y daño pulmonar (Csordas \& Bernhard, 2013).

\section{Impacto gastrointestinal}

Más allá del impacto respiratorio y cardiovascular del consumo de cigarrillo, las evidencias sugieren que el tabaquismo también es deletéreo para el sistema gastrointestinal. En este contexto, está demostrado que el consumo de cigarrillo aumenta la incidencia y la recurrencia de úlceras pépticas. Así también, el cigarrillo se asocia a Síndrome de Intestino Irritable, Enfermedad de Crohn y al desarrollo de cáncer de esófago, estómago, hígado, páncreas y colon (Wu \& Cho, 2004).

El impacto gastrointestinal del cigarrillo puede deberse en parte, a la gran cantidad de material particulado que es deglutido por el fumador. Está reportado que la concentración de nicotina en jugos gástricos es 10 veces superior a la concentración presente en sangre arterial, y 80 veces superior a la concentración en sangre venosa (Lindell et al., 1993).

Las evidencias sugieren que el consumo crónico de cigarrillo, y particularmente la nicotina, sería capaz de aumentar la secreción gástrica y reducir el pH estomacal (Li et al., 2014). Por otro lado, de acuerdo a algunos autores, el tabaquismo aumenta la probabilidad de infección por H. pylori, una bacteria altamente asociada al desarrollo de úlceras gastroduodenales y cáncer gástrico (Konturek et al., 2003). Si bien algunas investigaciones plantean lo contrario (Ogihara et al., 2000), los estudios sugieren que el consumo de cigarrillo exacerba la progresión de la enfermedad y empeora las tasas de erradicación (Nakamura et al., 2002). Por otro lado, el consumo crónico de cigarrillo pareciera alterar la producción de mucus por parte de la mucosa gástrica, junto con alterar el proceso de restitución de la mucosa gastrointestinal (Li et al., 2014). Sin embargo, algunos investigadores han obtenido resultados opuestos al evaluar el impacto del cigarrillo en modelos animales a corto plazo (Wong et al., 1986), sugiriendo que el efecto depende del modelo de estudio y principalmente del tiempo de exposición.

Por otro lado, el carácter vasoconstrictor y procoagulante del humo de cigarrillo también tiene impacto a nivel gastrointestinal. En este contexto, está demostrado que el consumo crónico de cigarrillo afecta la microcirculación y reduce significativamente el flujo sanguíneo hacia la mucosa gastrointestinal. Particularmente, los estudios demuestran que la nicotina puede inducir la liberación de 
vasopresina, la cual favorece la vasoconstricción y agregación plaquetaria, que sumado al efecto de la fracción oxidante en la función endotelial, alteran la irrigación del tejido (Hunsballe et al., 2001).

Con respecto a la inflamación intestinal, el papel del cigarrillo ha sido un foco de gran interés, debido al efecto paradojal observado en los pacientes con Enfermedad Inflamatoria Intestinal (EII). Las enfermedades inflamatorias intestinales comprenden un conjunto de desórdenes cuya característica principal es la presencia de inflamación crónica o recurrente en el tracto gastrointestinal, siendo las dos entidades más importantes, la Enfermedad de Crohn (EC) y la Colitis Ulcerosa (CU). Mientras el consumo de cigarrillo constituye el principal factor ambiental de riesgo para EC, es actualmente el factor epidemiológico más consistentemente asociado a una menor incidencia de CU (Cosnes, 2008). Este papel diferencial aún no ha logrado ser explicado y pareciera no solo depender de la diferencias etiológicas entre ambas patologías, sino también de un impacto sitio-específico, tal como será detallado en esta revisión.

\section{Impacto en ElI}

\section{Enfermedad inflamatoria Intestinal: diferencias entre EC y CU}

Las enfermedades inflamatorias intestinales son desórdenes crónicos que generalmente comienzan en la adultez temprana, acompañados de diarrea recurrente, dolor abdominal y sangre en deposiciones. Si bien en los últimos años ha existido un progreso importante en el conocimiento de estas enfermedades, la etiología aún no ha podido ser definida. Tanto la incidencia de la EC, como de la CU, han aumentado significativamente a nivel mundial, y aún no existe un tratamiento curativo disponible (Cosnes et al., 2011).

Durante las últimas décadas, los investigadores han concluido que los principales componentes responsables de la Ell serían los factores genéticos, el ambiente, la microbiota intestinal y la respuesta inmune (de Souza \& Fiocchi, 2016). En este contexto, diversos cambios en el estilo de vida, capaces de desregular la microbiota o el sistema inmune, podrían explicar el aumento en la incidencia de estas enfermedades, como por ejemplo, el uso de antibióticos, la dieta occidental y el tabaquismo.

Más allá de diversas características compartidas, ambos desórdenes pueden ser distinguidos por diferencias en las características clínicas, endoscópicas e histológicas (Ordás et al., 2012) y por algunos factores involucrados, como lo es el impacto del cigarrillo. La CU se caracteriza por ser un proceso inflamatorio continuo que compromete solo la mucosa del segmento distal del intestino, partiendo normalmente desde el recto y expandiéndose hacia el resto del colon (Ordás et al., 2012). A diferencia de esto, la EC puede abarcar desde la boca a la zona perianal, de forma discontinua y transmural. Dependiendo de la localización de la inflamación, la EC se ha dividido tradicionalmente en fenotipo ileal, colónico, ileo-colónico y con lesiones en el tracto gastrointestinal alto, siendo la inflamación ileal la más frecuente (Baumgart \& Sandborn, 2012). Si bien la afectación de colon suele ser más sintomática, la inflamación ileal pareciera progresar más rápidamente hacia lesiones transmurales, como fístulas o estenosis (Oberhuber et al., 2000).

La causa precisa de estas enfermedades aun es incierta, sin embargo, pareciera ser que en ambos casos, individuos genéticamente susceptibles tienen desregulada la respuesta inmune de la mucosa a su microbiota intestinal, lo cual desemboca en una inflamación del tracto digestivo. Por lo mismo, en los últimos años las investigaciones han dejado de lado el foco inicial que se había concentrado en la respuesta inmune adaptativa. Sin embargo, es sabido que la respuesta inmune adaptativa es fundamental para perpetuar la inflamación, e interesantemente, su modo de participación diferiría entre ambas entidades. Mientras la EC ha sido clasificada históricamente como un desorden inmunológico con exacerbación del fenotipo Th1/Th17, la CU se caracteriza por una respuesta atípica Th2 (de Souza \& Fiocchi, 2016). Por otro lado, diversos estudios han sugerido que la respuesta inmune adaptativa pudiera tener un papel etiológico más importante en CU que en EC. Incluso, algunos estudios han reportado que tanto el daño intestinal como las manifestaciones extraintestinales presentes en los pacientes con CU, podrían deberse a una respuesta inmune adaptativa mediada por anticuerpos contra autoantígenos (Takahashi \& Das, 1985).

A diferencia de esto, los pacientes con EC presentan una mayor propensión a desarrollar anticuerpos contra antígenos bacterianos, sugiriendo que en este caso la respuesta humoral pareciera ser más bien el resultado de una alteración a nivel de la barrera de la mucosa y de una mayor interacción con la microbiota intestinal (Man et al., 2011). De hecho, el rol fundamental de la disrupción del epitelio gastrointestinal y de los microorganismos en la patogénesis de la EC, ha sido ampliamente demostrada, tanto en humanos como modelos animales (Man et al., 2011).

Interesantemente, estas aparentes diferencias fisiopatológicas son importantes para tratar de explicar el impacto diferencial del cigarrillo. 


\section{Efectos opuestos del cigarrillo en EC y CU: epidemiología}

Actualmente ha sido ampliamente aceptado que el consumo de cigarrillo protege contra CU. Un meta-análisis confirmó que el riesgo de desarrollar CU en personas con hábito tabáquico es significativamente menor en comparación al riesgo de personas que nunca han fumado (OR 0.58 [0.45-0.75]) (Mahid et al., 2006). Además, las tasas de hospitalización, recurrencia, necesidad de tratamientos más agresivos y colectomías, son significativamente menores en pacientes fumadores (Cosnes, 2008).

El efecto del consumo de cigarrillo en CU es temporal, ya que el riesgo relativo de desarrollar CU no está disminuido en individuos ex-fumadores. Es más, dejar de fumar aumenta el riesgo de desarrollar CU en comparación a los individuos que nunca han fumado (OR 1.64 [1.36-1.98]) (Calkins, 1989), y aumenta la actividad de la enfermedad (Beaugerie et al., 2001).

Por el contrario, está demostrado que el tabaquismo temprano aumenta significativamente el riesgo de desarrollar EC (OR 2.0 [1.65-2.47]) (Calkins, 1989). Además, los pacientes con EC fumadores presentan un peor curso de la enfermedad y una peor calidad de vida, con mayor riesgo de complicaciones, mayor tasa de hospitalizaciones, peor respuesta a tratamientos y mayor necesidad de intervenciones quirúrgicas (Cosnes et al., 1999). Interesantemente, existe una clara asociación entre el consumo de cigarrillo y la localización de la inflamación en estos pacientes, radicándose particularmente a nivel de íleon (fenotipo ileal) (Brant et al., 2003), lo cual ha sido confirmado en modelos animales (Zuo et al., 2014). En estos pacientes además, las tasas de recurrencia clínica y quirúrgica son significativamente mayores a la de pacientes no fumadores. De hecho, un 70\% de los pacientes con EC fumadores que han debido ser sometidos a resección ileal, sufren lesiones macroscópicas en el sitio de la anastomosis al primer año tras la cirugía, en comparación de un 35\% de los pacientes no fumadores (Yamamoto \& Keighley, 2000).

Al igual que en el caso de la CU, el impacto del consumo de cigarrillo en EC es temporal, y el dejar de fumar mejora el curso de la enfermedad. De hecho, se ha estimado que tras 2 años de dejar de fumar, la actividad de la enfermedad y el requerimiento terapéutico de los ex-fumadores se iguala al de los pacientes que nunca han fumado (Cosnes et al., 2001; Lewis et al., 2007).

\section{Efectos opuestos del cigarrillo en EC y CU: mecanismos propuestos}

Los mecanismos por los cuales el cigarrillo puede actuar de forma tan diferente entre ambas entidades es difícil de definir, debido a la gran cantidad de componentes presentes en el humo de cigarrillo y debido a la gran cantidad de factores que puede estar alterando, incluido la barrera mucosa, la microbiota, el sistema inmune, la microvasculatura, etc. Particularmente llama la atención que pese al efecto deletéreo del cigarrillo en el tracto gastrointestinal, el tabaquismo protege contra CU. En este contexto se han propuesto diversas propiedades farmacológicas para el humo de cigarrillo.

En primer lugar, estudios han demostrado que la nicotina tendría un efecto inmunomodulador mediado por la activación de receptores nicotínicos a7 (a7-nAChR) en células inmunes, tales como macrófagos y células dendríticas. Específicamente, está demostrado que la estimulación de estos receptores en células inmunes reduce la producción de IL-1 $\beta$, IL-2, IL-8 y TNF-a, tanto in vitro como in vivo (Lakhan \& Kirchgessner, 2011). Evidencias adicionales sugieren que la activación de los receptores a7-nAChR es un factor crítico en la función inmunosupresora de células T reguladoras CD4(+) CD25(+), reduciendo la activación de NF-KB y la producción de IL-2 (Lakhan \& Kirchgessner, 2011). Interesantemente, otros estudios han demostrado que este receptor también se expresa en células endoteliales, cuya estimulación reduce la producción de quimioquinas y la expresión de moléculas de adhesión por el endotelio, mecanismo por el cual también podría ayudar a modular la migración leucocitaria y la inflamación (Saeed et al., 2005). Por otro lado, al monóxido de carbono (CO), principal componente de la fase gaseosa del humo de cigarrillo, también se le han atribuido propiedades antiinflamatorias. Está demostrado que el CO es capaz de inhibir la maduración de células dendríticas y de reducir la presentación de antígenos. Además, está reportado que el CO reduce la producción de citoquinas proinflamatorias y la proliferación de linfocitos T efectores, mientras que estimula la secreción de la citoquina antiinflamatoria IL-10 (Mackern-Oberti et al., 2014). Es más, la exposición a CO ha mostrado generar mejorías en modelos murinos genéticos y químicos de Colitis Ulcerosa (Mackern-Oberti et al., 2014; Sheikh et al., 2011).

En contraposición a estos efectos favorables observados en $\mathrm{CU}$, en la EC ileal prevalecen los efectos negativos del cigarrillo. Considerando que parte del proceso de la EC es el desarrollo de microinfartos multifocales a nivel gastrointestinal, es razonable pensar que el cigarrillo puede agravar la enfermedad al empeorar la función endotelial. Está demostrado que el conjunto de componentes oxidantes y altamente reactivos del cigarrillo pueden amplificar la alteración en la capacidad de vasodilatación de una microvasculatura crónicamente inflamada, resultando en isquemia, ulceraciones y fibrosis (Jaimes et al., 2004). Por otro lado, el daño oxidativo provocado por el consumo de cigarrillo también causa alteraciones a nivel de lípidos, proteínas, DNA e incluso organelos, que eventualmente deberán ser solucionadas mediante un proceso de autofagia, para evitar la muerte celular. Considerando que la 
autofagia pareciera ser un proceso indispensable para la respuesta inmune contra patógenos, un requerimiento exhaustivo de autofagia causado por los componentes del cigarrillo, pudiera ayudar a explicar el mayor riesgo a desarrollar EC, especialmente en individuos genéticamente susceptibles (Verschuere et al., 2011). Más aún, el consumo de cigarrillo y particularmente la nicotina, pueden alterar la interacción de la mucosa intestinal con la microbiota por diversos mecanismos. En primer lugar, al reducir la activación de macrófagos, la nicotina puede afectar el clearance bacteriano. Así también, está demostrado que el consumo de cigarrillo altera significativamente la microbiota intestinal, pudiendo favorecer la presencia de patógenos oportunistas, como enterobacterias y algunas especies de bacteroidetes (Biedermann et al., 2014). Un estudio demostró que el impacto del tabaquismo en la microbiota intestinal es temporal, y que a los meses de cesar el consumo, disminuye la abundancia de enterobacterias y bacteroidetes, mientras aumenta la abundancia de algunos firmicutes (Biedermann et al., 2014). Dado que en los últimos diez años ha sido ampliamente aceptado que el desequilibrio en la microbiota intestinal es uno de los principales contribuyentes en la fisiopatología de ambas entidades de Ell, el impacto del cigarrillo en la microbiota resulta un tema de particular interés.

\section{Efectos opuestos del cigarrillo en EC y CU: ¿diferencias etiológicas o impacto sitio-específico?}

Si bien, todos los mecanismos antes mencionados pueden ayudar a explicar el impacto del cigarrillo en la CU y la EC, no logran explicar el efecto diferencial del tabaquismo en la inflamación intestinal. En este contexto, hay tres hipótesis posibles, no excluyentes entre sí, que serán descritas a continuación.

En primer lugar, el impacto diferencial del cigarrillo en EC ileal y CU pudiera depender de las diferencias etiológicas entre ambas entidades. Tal como fue mencionado, en la etiología de la EC pareciera ser crucial la disrupción de la mucosa intestinal y su interacción con una microbiota alterada. En este contexto, un estudio demostró que ratones expuestos a humo de cigarrillo presentan daños a nivel de la barrera de la mucosa de íleon, provocando una mayor permeabilidad a bacterias (Zuo et al., 2014). Así también, es factible que la alteración causada por el cigarrillo en la microbiota intestinal tenga un impacto más significativo en la EC. A diferencia de esto, el sistema inmune adaptativo pareciera jugar un papel más relevante en el desarrollo y progresión de la CU. Por lo mismo, el efecto inmunomodulador sistémico reportado para la nicotina y el $\mathrm{CO}$, pudiera prevalecer en la CU por sobre los impactos deletéreos del cigarrillo en la mucosa intestinal, reportados en EC.
Por otro lado, el impacto diferencial del cigarrillo de acuerdo con la localización de la inflamación, pareciera apuntar a un efecto sitio específico. De hecho, estudios en modelos murinos han demostrado que la exposición al cigarrillo exacerba el daño en intestino delgado (Zuo et al., 2014), mientras que reduce la colitis (Eliakim et al., 2002). Este impacto sitio específico puede depender de una llegada diferencial de los componentes del cigarrillo a los distintos tejidos, o más bien de un mecanismo tejido-dependiente, constituyendo así las dos hipótesis restantes.

Los componentes volátiles del humo son inhalados y rápidamente absorbidos por mucosas y alvéolos, donde alcanzan la circulación. En cambio, un alto porcentaje del material particulado es deglutido, y alcanza grandes concentraciones en saliva y jugos gástricos. En estos últimos, diversos componentes derivados del tabaco de carácter alcalino se concentran debido al $\mathrm{pH}$, ya que no pueden ser absorbidos en su forma ionizada (Lindell et al., 1993). Posteriormente, estos componentes alcanzarán el intestino delgado, donde podrán ser absorbidos a medida que aumenta el $\mathrm{pH}$ hacia la zona distal, particularmente en el íleon. Por lo tanto, como segunda hipótesis, el gran impacto del humo de cigarrillo a nivel de la mucosa ileal, puede ser atribuido a la alta exposición de esa zona a los componentes del cigarrillo. De hecho, es posible que la parte distal del intestino grueso se vea mayoritariamente influida por los componentes circulantes, donde tanto el monóxido de carbono como la nicotina pueden modular el perfil inflamatorio y la migración leucocitaria, prevaleciendo el papel inmunomodulador por sobre el impacto en la mucosa. En conjunto a esto, y como tercera hipótesis, el impacto nocivo del cigarrillo en el íleon podría ser atribuido a un efecto tejido-dependiente. En este contexto, son diversos los factores propios del íleon que pueden verse afectados por los componentes del cigarrillo, como por ejemplo, placas de Peyer, células de Paneth y la microbiota específica. Interesantemente, tanto las células inmunes presentes en placas de Peyer, como las células de Paneth, son altamente dependientes del proceso de autofagia, por lo que podrían verse severamente afectadas por los componentes reactivos del cigarrillo. De hecho, un estudio demostró que la exposición de ratones a humo de cigarrillo aumenta significativamente el proceso de autofagia en Placas de Peyer y en el epitelio asociado a estas (Verschuere et al., 2011), pero aún falta por dilucidar el impacto real de este efecto en los pacientes.

En conclusión, son diversos los mecanismos por los cuales el cigarrillo puede afectar la inflamación intestinal, donde posiblemente las tres hipótesis mencionadas no sean excluyentes, sino más bien complementarias. Al tratarse de patologías multifactoriales, 
probablemente este factor pueda afectar en diferente magnitud en cada individuo, donde el resultado final estará modulado por componentes genéticos, localización y actividad de la enfermedad, frecuencia del consumo y otros factores ambientales.

\section{Agradecimientos}

Trabajo financiado por FONDECYT 1131012 y Beca CONICYT Doctorado Nacional 21140273. Los autores declaran no tener conflictos de interés.

\section{Referencias}

Baumgart, D.C., \& Sandborn, W.J. (2012). Crohn's disease. Lancet 380, 1590-1605.

Beaugerie, L., Massot, N., Carbonnel, F., Cattan, S., Gendre, J.P., \& Cosnes, J. (2001). Impact of cessation of smoking on the course of ulcerative colitis. Am. J. Gastroenterol. 96, 2113-2116.

Biedermann, L., Brülisauer, K., Zeitz, J., Frei, P., Scharl, M., Vavricka, S.R., Fried, M., Loessner, M.J., Rogler, G., \& Schuppler, M. (2014). Smoking cessation alters intestinal microbiota: insights from quantitative investigations on human fecal samples using $\mathrm{FISH}$. Inflamm. Bowel Dis. 20, 1496-1501.

Brant, S.R., Picco, M.F., Achkar, J.-P., Bayless, T.M., Kane, S. V, Brzezinski, A., Nouvet, F.J., Bonen, D., Karban, A., Dassopoulos, T., et al. (2003). Defining complex contributions of NOD2/CARD15 gene mutations, age at onset, and tobacco use on Crohn's disease phenotypes. Inflamm. Bowel Dis. 9, 281-289.

Brusselle, G.G., Joos, G.F., \& Bracke, K.R. (2011). New insights into the immunology of chronic obstructive pulmonary disease. Lancet (London, England) 378, 1015-1026.

Calafat, A.M., Polzin, G.M., Saylor, J., Richter, P., Ashley, D.L., \& Watson, C.H. (2004). Determination of tar, nicotine, and carbon monoxide yields in the mainstream smoke of selected international cigarettes. Tob. Control 13, 45-51.

Calkins, B.M. (1989). A meta-analysis of the role of smoking in inflammatory bowel disease. Dig. Dis. Sci. 34, 1841-1854.

Cosnes, J. (2008). What is the link between the use of tobacco and IBD? Inflamm. Bowel Dis. 14 Suppl 2, S14-S15.

Cosnes, J., Carbonnel, F., Carrat, F., Beaugerie, L., Cattan, S., \& Gendre, J. (1999). Effects of current and former cigarette smoking on the clinical course of Crohn's disease. Aliment. Pharmacol. Ther. 13, 1403-1411.
Cosnes, J., Beaugerie, L., Carbonnel, F., \& Gendre, J.P. (2001). Smoking cessation and the course of Crohn's disease: an intervention study. Gastroenterology 120, 1093-1099.

Cosnes, J., Gower-Rousseau, C., Seksik, P., \& Cortot, A. (2011). Epidemiology and natural history of inflammatory bowel diseases. Gastroenterology 140, 1785-1794.

Csordas, A., \& Bernhard, D. (2013). The biology behind the atherothrombotic effects of cigarette smoke. Nat. Rev. Cardiol. 10, 219-230.

De Souza, H.S.P., \& Fiocchi, C. (2016). Immunopathogenesis of IBD: current state of the art. Nat. Rev. Gastroenterol. Hepatol. 13, 13-27.

Eliakim, R., Fan, Q.X., \& Babyatsky, M.W. (2002). Chronic nicotine administration differentially alters jejunal and colonic inflammation in interleukin-10 deficient mice. Eur.J. Gastroenterol. Hepatol. 14, 607-614.

Hunsballe, J.M., Rittig, S., Pedersen, E.B., \& Djurhuus, J.C. (2001). Smokeless nicotinergic stimulation of vasopressin secretion in patients with persisting nocturnal enuresis and controls. Scand. J. Urol. Nephrol. 35, 117-121.

Jaimes, E.A., DeMaster, E.G., Tian, R.-X., \& Raij, L. (2004). Stable compounds of cigarette smoke induce endothelial superoxide anion production via NADPH oxidase activation. Arterioscler. Thromb. Vasc. Biol. 24, 1031-1036.

Konturek, S.J., Bielański, W., Płonka, M., Pawlik, T., Pepera, J., Konturek, P.C., Czarnecki, J., Penar, A., \& Jedrychowski, W. (2003). Helicobacter pylori, non-steroidal anti-inflammatory drugs and smoking in risk pattern of gastroduodenal ulcers. Scand. J. Gastroenterol. 38, 923-930.

Lakhan, S.E., \& Kirchgessner, A. (2011). Anti-inflammatory effects of nicotine in obesity and ulcerative colitis. J. Transl. Med. 9, 129.

Lewis, C.M., Whitwell, S.C.L., Forbes, A., Sanderson, J., Mathew, C.G., \& Marteau, T.M. (2007). Estimating risks of common complex diseases across genetic and environmental factors: the example of Crohn disease. J. Med. Genet. 44, 689-694.

Li, L.F., Chan, R.L.Y., Lu, L., Shen, J., Zhang, L., Wu, W.K.K., Wang, L., Hu, T., Li, M.X., \& Cho, C.H. (2014). Cigarette smoking and gastrointestinal diseases: the causal relationship and underlying molecular mechanisms (review). Int. J. Mol. Med. 34, 372-380.

Lindell, G., Farnebo, L.O., Chen, D., Nexø, E., Rask Madsen, J., Bukhave, K., \& Graffner, H. (1993). Acute effects of smoking during modified sham feeding in duodenal ulcer patients. An analysis of nicotine, acid secretion, gastrin, catecholamines, epidermal growth factor, prostaglandin E2, and bile acids. Scand. J. Gastroenterol. 28, 487-494. 
Mackern-Oberti, J.P., Riquelme, S.A., Llanos, C., Schmidt, C.B., Simon, T., Anegon, I., Jara, E., Riedel, C.A., Bueno, S.M., \& Kalergis, A.M. (2014). Heme oxygenase-1 as a target for the design of gene and pharmaceutical therapies for autoimmune diseases. Curr. Gene Ther. 14, 218-235.

Mahid, S.S., Minor, K.S., Soto, R.E., Hornung, C.A., \& Galandiuk, S. (2006). Smoking and inflammatory bowel disease: a meta-analysis. Mayo Clin. Proc. 81, 1462-1471.

Man, S.M., Kaakoush, N.O., \& Mitchell, H.M. (2011). The role of bacteria and pattern-recognition receptors in Crohn's disease. Nat. Rev. Gastroenterol. Hepatol. 8, 152-168.

Marcilla, A., Martínez, I., Berenguer, D., Gómez-Siurana, A., \& Beltrán, M.I. (2012). Comparative study of the main characteristics and composition of the mainstream smoke of ten cigarette brands sold in Spain. Food Chem. Toxicol. 50, 1317-1333.

Nakamura, M., Haruma, K., Kamada, T., Mihara, M., Yoshihara, M., Sumioka, M., Fukuhara, T., \& Chayama, K. (2002). Cigarette smoking promotes atrophic gastritis in Helicobacter pylori-positive subjects. Dig. Dis. Sci. 47, 675-681.

Oberhuber, G., Stangl, P.C., Vogelsang, H., Schober, E., Herbst, F., \& Gasche, C. (2000). Significant association of strictures and internal fistula formation in Crohn's disease. Virchows Arch. 437, 293-297.

Ogihara, A., Kikuchi, S., Hasegawa, A., Kurosawa, M., Miki, K., Kaneko, E., \& Mizukoshi, H. (2000). Relationship between Helicobacter pylori infection and smoking and drinking habits. J. Gastroenterol. Hepatol. 15, 271-276.

Ordás, I., Eckmann, L., Talamini, M., Baumgart, D.C., \& Sandborn, W.J. (2012). Ulcerative colitis. Lancet (London, England) 380, 1606-1619.

Organización Mundial de la Salud (2015). OMS | Tabaco (http:// www.who.int/mediacentre/factsheets/fs339/es/).

Saeed, R.W., Varma, S., Peng-Nemeroff, T., Sherry, B., Balakhaneh, D., Huston, J., Tracey, K.J., Al-Abed, Y., \& Metz, C.N. (2005). Cholinergic stimulation blocks endothelial cell activation and leukocyte recruitment during inflammation. J. Exp. Med. 201, 1113-1123.
Sheikh, S.Z., Hegazi, R.A., Kobayashi, T., Onyiah, J.C., Russo, S.M., Matsuoka, K., Sepulveda, A.R., Li, F., Otterbein, L.E., \& Plevy, S.E. (2011). An anti-inflammatory role for carbon monoxide and heme oxygenase-1 in chronic Th2-mediated murine colitis. J. Immunol. 186, 5506-5513.

Takahashi, F., \& Das, K.M. (1985). Isolation and characterization of a colonic autoantigen specifically recognized by colon tissue-bound immunoglobulin G from idiopathic ulcerative colitis. J. Clin. Invest. 76, 311-318.

Verschuere, S., Bracke, K.R., Demoor, T., Plantinga, M., Verbrugghe, P., Ferdinande, L., Lambrecht, B.N., Brusselle, G.G.G., \& Cuvelier, C.A. (2011). Cigarette smoking alters epithelial apoptosis and immune composition in murine GALT. Lab. Invest. 91, 1056-1067.

Wong, S.H., Ogle, C.W., \& Cho, C.H. (1986). The influence of chronic or acute nicotine pretreatment on ethanol-induced gastric ulceration in the rat. J. Pharm. Pharmacol. 38, 537-540.

Wu, W.K.K., \& Cho, C.H. (2004). The pharmacological actions of nicotine on the gastrointestinal tract. J. Pharmacol. Sci. 94, 348-358.

Yamamoto, T., \& Keighley, M.R. (2000). Smoking and disease recurrence after operation for Crohn's disease. Br. J. Surg. 87, 398-404.

Yu, K.-N., Kim, H.-J., Kim, S., Dawaadamdin, O., Lee, A.-Y., Hong, S.-H., Chang, S.-H., Choi, S.-J., Shim, S.-M., Lee, K., et al. (2016). Cigarette Smoking Condensate Disrupts Endoplasmic Reticulum-Golgi $\mathrm{Ne}$ twork Homeostasis Through GOLPH3 Expression in Normal Lung Epithelial Cells. Nicotine Tob. Res. 18, 1877-1885.

Zuo, L., Li, Y., Wang, H., Wu, R., Zhu, W., Zhang, W., Cao, L., Gu, L., Gong, J., Li, N., et all. (2014). Cigarette smoking is associated with intestinal barrier dysfunction in the small intestine but not in the large intestine of mice. J. Crohns. Colitis 8, 1710-1722.

Van Zyl-Smit, R.N., Brunet, L., Pai, M., \& Yew, W.-W. (2010). The convergence of the global smoking, COPD, tuberculosis, HIV, and respiratory infection epidemics. Infect. Dis. Clin. North Am. 24, 693-703. 